\title{
COMPORTAMENTO DOS ÓLEOS DE GIRASSOL, SOJA E MILHO EM FRITURAS DE PRODUTO CÁRNEO EMPANADO PRÉ-FRITO CONGELADO
}

\author{
Sunflower, soybean and corn oils behavior in frozen pre-fried coated \\ meat product frying
}

\author{
Patrícia Vieira Del Ré ${ }^{1}$, Neuza Jorge ${ }^{2}$
}

\begin{abstract}
RESUMO
A utilização crescente de alimentos rápidos determinou a expansão de uma indústria de produtos fritos e pré-fritos. A compreensão das mudanças que o óleo sofre durante os processos de fritura é importante, pois pode levar à otimização destes processos, e a melhoria da qualidade do óleo de fritura e do produto final. Neste trabalho, objetivou-se determinar os níveis de alteração de diferentes óleos vegetais, girassol, soja e milho, no processo de fritura dos snacks - produto cárneo empanado pré-frito congelado. As frituras foram conduzidas em temperatura de $180^{\circ} \mathrm{C}$, relação superfície/volume $(\mathrm{S} / \mathrm{V}) \mathrm{de} 0,3 \mathrm{~cm}^{-1} \mathrm{e}_{\text {empo total de }}$ aquecimento de 12 horas. Nos snacks procederam-se as análises de teor de umidade e lipídios; nos óleos as determinações de compostos polares totais, ácidos graxos livres e índice de peróxidos. Nenhuma análise apresentou alteração acima dos limites recomendados por alguns países. Desta forma, concluiu-se que as condições estabelecidas no processo de fritura são seguras, indicando que todos os óleos foram adequados à fritura, sendo o óleo de milho de maior estabilidade oxidativa.
\end{abstract}

Termos para indexação: Óleos vegetais, fritura, compostos polares totais.

\section{ABSTRACT}

The ever growing utilization of fast food determined the expansion of an industry of pre-fried and fried products. The understanding of the changes that the oil undergoes during the frying processes is outstanding because it may lead to the optimization of such processes as well as to improvements on the quality of both frying oil and the finished product. The purpose of the present study was to determine the alteration levels of different vegetable oils, sunflower, soybean and corn, in the frying process of snacks - frozen pre-fried meat coated product. The frying sessions were carried out at $180^{\circ} \mathrm{C}$ temperature, $0.3 \mathrm{~cm}^{-1}$ surface/volume ratio and 12 hours overall heating time. As for the snacks, the moisture rate and lipids analyses were performed; for the oils, total polar compounds, free fatty acids and peroxide indices were determined. No analysis presented any alteration above the limits recommended by some countries. Therefore, it was concluded that the conditions established in the frying process are safe, suggesting that all of the oils were fit for the frying, being the corn oil the one of the highest oxidative stability.

Index terms: Vegetable oils, frying, total polar compounds.

(Recebido em 9 de março de 2006 e aprovado em 10 de abril de 2007)

\section{INTRODUÇÃO}

O crescimento de diversos setores de consumo de óleos se deve à modificação dos hábitos alimentares, resultado de uma combinação de profundas mudanças sociais, econômicas e tecnológicas, associados ao grande desenvolvimento dos setores de restaurantes, sistemas de alimentação coletiva e alimentos de consumo imediato (POZO-DÍEZ, 1995).

A aceitação de alimentos processados por fritura é universal e apreciada por diferentes grupos populacionais. As diversas mudanças registradas nas atitudes do consumidor ao longo dos últimos anos fizeram com que as indústrias de alimentos passassem a dispor de produtos específicos para o processo de fritura, como os alimentos congelados, que são largamente aceitos pelo mercado consumidor (CORSINI \& JORGE, 2006).

$\mathrm{Na}$ ingestão de alimentos fritos destacam-se dois aspectos nutricionais importantes: a contribuição da fritura na ingestão total de lipídios na dieta e, o impacto da ingestão de substâncias potencialmente tóxicas formadas durante o tratamento térmico, em destaque os monômeros cíclicos, compostos nutricionalmente indesejável.

O processo de fritura incorpora o óleo do processo aos lipídeos totais do alimento tornando-o, assim, uma fonte mais concentrada de energia. Esse fato isolado é benéfico, considerando a qualidade sensorial do alimento, porém pode desencadear um maior consumo de calorias e,

${ }^{1}$ Mestre em Engenharia e Ciência de Alimentos - Departamento de Engenharia e Tecnologia de Alimentos/DETA - Universidade Estadual Paulista/UNESP _ Rua Cristóvão Colombo, 2265, Jardim Nazareth - 15054-000 - São José do Rio Preto, SP - delre@unirpnet.com.br

2Doutora, Professora Assistente - Departamento de Engenharia e Tecnologia de Alimentos/DETA - Universidade Estadual Paulista/UNESP - Rua Cristóvão Colombo, 2265, Jardim Nazareth - 15054-000 - São José do Rio Preto, SP - njorge@ibilce.unesp.br 
conseqüentemente, no aumento do peso corpóreo, que sob alguns aspectos pode ser indesejável (BERGER, 1984).

Muitos alimentos, antes de serem submetidos ao processo de fritura, são empanados com farinha, funcionando como uma barreira protetora, de tal forma a reduzir a perda de peso e manter o produto frito mais macio e mais saboroso. Segundo Pokorny (1998), a evaporação de água do alimento durante a operação de fritura significa uma perda substancial de peso que é particularmente importante no caso de produtos cárneos fritos, visto que se trata de um produto de custo elevado.

O óleo, durante o processo de fritura, interage com o ar, água e componentes dos alimentos que estão sendo fritos, gerando reações de oxidação e degradação dos óleos (DAMY \& JORGE, 2003).

A avaliação da alteração e a identificação dos compostos que são formados durante a fritura de alimentos é de grande importância e interesse, não só para pesquisadores, como também para consumidores, indústrias de alimentos e administrações ligadas à saúde pública.

Embora o Brasil não possua legislação para a utilização de óleos de fritura, alguns países, tais como Alemanha, Bélgica, Holanda, Estados Unidos, Espanha, Suíça, França, Japão e Chile, possuem leis e regulamentações de controle de qualidade de óleos de fritura que visam garantir a qualidade dos mesmos e dos alimentos fritos (FIRESTONE, 1993).

Conhecer os compostos alterados formados durante o processo de fritura, definir os óleos e/ou gorduras mais idôneos para fritura e estabelecer o momento em que estes devem ser descartados, são questões que têm refletido em um aumento de estudos nesta área, pois tem impacto econômico e pode implicar em redução final dos custos e controle da qualidade do alimento. Neste trabalho objetivou-se determinar os níveis de alteração de diferentes óleos vegetais, girassol, soja e milho, no processo de fritura dos snacks - produto cárneo empanado pré-frito congelado.

\section{MATERIAL E MÉTODOS}

\section{Processo de fritura}

Foram utilizados nos testes de frituras descontínuas os seguintes óleos vegetais: óleo de girassol refinado (OGR); óleo de soja refinado (OSR); óleo de milho refinado (OMR); provenientes do comércio local de São José do Rio Preto.

Os snacks - produto cárneo empanado pré-frito congelado (Big Chichen Tradicional), utilizados para as frituras foram obtidos de empresa registrada no SIF n.1001.
Os ensaios de fritura descontínua foram conduzidos em fritadeira doméstica comercial, com capacidade de 3 litros. Durante o processo de fritura foram controladas as seguintes condições: temperatura de $180 \pm 2^{\circ} \mathrm{C}$, relação S/ $\mathrm{V}$ de $0,3 \mathrm{~cm}^{-1}$ e tempo total de experimento de 12 horas.

Inicialmente foram colocados três litros de óleo na fritadeira, os quais foram aquecidos por um período de 10 minutos. Cada lote de produto foi frito por 5 minutos, empregando um intervalo de 30 minutos entre cada fritura, sendo que 5 minutos foram utilizados para o reaquecimento do óleo antes de iniciar a fritura seguinte. Foram realizadas as frituras de 25 lotes, cada lote com aproximadamente 500 $\mathrm{g}$ de produto. Foi empregada reposição de óleo novo nos intervalos das frituras para manter a relação $\mathrm{S} / \mathrm{V}$ constante, correspondendo a uma média de $820 \mathrm{~mL}$, em cada processo de fritura. O período de experimento para cada óleo foi de 2 horas, divididos em dois dias consecutivos de frituras descontínuas, sendo 6 horas em cada dia. Foi empregada a filtragem do óleo após as 6 horas iniciais.

As amostras de óleo e produtos foram coletadas nos tempos 0,$5 ; 2,5 ; 5,0 ; 7,0 ; 9,5$ e 12 horas de fritura. As amostras de óleos $(80 \mathrm{~mL})$ foram coletadas em recipientes de vidro âmbar e os produtos (100 g) foram embalados em folha de alumínio e, em seguida, armazenados à temperatura de aproximadamente $-20^{\circ} \mathrm{C}$. As amostras foram descongeladas no momento das análises, as quais foram realizadas em duplicatas.

\section{Determinações analíticas}

O teor de umidade foi obtido pelo método Bc 2-49 (AOCS, 1990), expresso em porcentagem, através da relação entre o peso de água eliminada do produto pelo peso da amostra total; o teor lipídico pelo método Bc 3-49 (AOCS, 1993), expresso em porcentagem, determinado no equipamento de extração de gordura, modelo MA 490 da marca Marconi. Os compostos polares foram determinados pelo método cromatográfico, proposto por Dobarganes et al. (2000b), expressos em porcentagem. Os ácidos graxos livres foram determinados pelo método Cd 3d-63 (AOCS, 1993), expressos em porcentagem de ácido oléico e o índice de peróxidos pelo método proposto pela Cd 8-53 (AOCS, 1993), expressos em milequivalentes de oxigênio ativo por $\mathrm{kg}$ de óleo.

\section{Análise estatística}

Para as determinações analíticas foram estabelecidos os seguintes fatores: Óleos (OGR, OSR e OMR) e Tempos de Fritura ( 0,$5 ; 2,5 ; 5,0 ; 7,0 ; 9,5$ e 12 horas). $\mathrm{O}$ experimento foi realizado em um esquema fatorial $3 \times 6$, no delineamento inteiramente casualizado. 
Os resultados obtidos das determinações analíticas, em duplicata, foram submetidos à análise de variância e as diferenças entre as médias foram testadas a 1 e $5 \%$ de probabilidade, pelo teste de Tukey, através do programa ESTAT - Sistema para Análises Estatísticas, versão 2.0, 1999, Brasil.

\section{RESULTADOS E DISCUSSÃO}

Os snacks utilizados continham, originalmente 54,21 e $32,46 \%$ de umidade e lipídios, respectivamente. Após as frituras, as médias desses percentuais variaram de 45,81 a $53,23 \%$ (média geral de $49,17 \%$ ) de umidade e 32,04 a $35,95 \%$ (média geral de 34,17\%), independentemente do tipo de óleo e tempo de fritura.

A perda de umidade dos snacks foi de $9,30 \%$, e a incorporação de lipídeos totais foi de 5,27\%. A baixa perda de umidade pode ser justificada, possivelmente pelo uso da técnica culinária de empanar o produto cárneo, prevenindo a perda de água.

Makinson et al. (1997) demostraram que alimentos com alto conteúdo de água e baixo teor de gordura estimulam a absorção de óleo; enquanto, alimentos de elevado conteúdo inicial de gordura não absorvem muito óleo durante a fritura; por outro lado, a gordura do alimento é transferida para o óleo do banho.

Para avaliar os efeitos dos fatores Óleos e Tempos de Fritura sobre o teor de umidade e teor lipídico do produto frito; compostos polares, ácidos graxos livres e índice de peróxidos nos óleos de fritura, foram realizados os cálculos da análise de variância e o teste $\mathrm{F}$ foi significativo $(\mathrm{P}<$
0,01) para a interação Óleos x Tempos de Fritura. Então, os efeitos dos diferentes óleos dependem dos tempos de fritura utilizados e os efeitos dos tempos de fritura, dependem dos óleos utilizados. Dessa forma, procedeu-se o desdobramento dessa interação e os resultados encontram-se nas Tabelas 1 e 2, respectivamente.

Em relação aos tempos de fritura, os snacks tiveram um comportamento instável (Tabela 1). Para todos os tipos de óleo houve oscilação entre aumento e diminuição do teor de umidade. Em relação ao tipo de óleo, verifica-se que com 0,5 hora de fritura, os valores do teor de umidade dos snacks não diferiram entre si para os três tipos de óleos estudados.

Observa-se aumento do teor de lipídico nos tempos intermediários das frituras, seguido por um decréscimo no último tempo de fritura, independentemente do tipo de óleo. De um modo geral apesar dos óleos diferirem significativamente ao longo do processo de fritura, os valores do teor lipídico foram próximos, proporcionando produtos com valores calóricos muitos similiares.

De acordo com Paul \& Mittal (1997), vários fatores afetam a penetração do óleo no alimento, como: forma geométrica do alimento, viscosidade do óleo de fritura, tipo de alimento, temperatura do meio de fritura e tempo de fritura, entre outros.

Em geral, a absorção ou incorporação de óleos depende mais da qualidade inicial do óleo de fritura do que do tipo de óleo ou gordura utilizada para fritar. A influência da qualidade do óleo é atribuída à formação de compostos de degradação, os quais aumentam a polaridade do meio

Tabela 1 - Médias de Teor de Umidade e Teor Lipídico para cada combinação de Óleos e Tempos de Fritura.

\begin{tabular}{|c|c|c|c|c|c|c|}
\hline \multirow[t]{2}{*}{ Óleos } & \multicolumn{6}{|c|}{ Tempos de Fritura (horas) } \\
\hline & 0,5 & 2,5 & 5,0 & 7,0 & 9,5 & 12,0 \\
\hline \multicolumn{7}{|c|}{ Teor de Umidade $(\%)$} \\
\hline Girassol & $48,86^{\mathrm{cA}}$ & $45,81^{\mathrm{dB}}$ & $49,25^{\mathrm{bcA}}$ & $50,51^{\mathrm{aA}}$ & $49,88^{\mathrm{abcB}}$ & $50,23^{\mathrm{abB}}$ \\
\hline Soja & $48,75^{\mathrm{bcA}}$ & $49,57^{\mathrm{bA}}$ & $47,44^{\mathrm{deB}}$ & $48,50^{\mathrm{cdC}}$ & $51,10^{\mathrm{aA}}$ & $46,49^{\mathrm{eC}}$ \\
\hline Milho & $48,14^{\mathrm{cA}}$ & $49,58^{\mathrm{bA}}$ & $49,34^{\mathrm{bA}}$ & $49,63^{\mathrm{bB}}$ & $48,85^{\mathrm{bcC}}$ & $53,23^{\mathrm{aA}}$ \\
\hline \multicolumn{7}{|c|}{ Teor Lipídico (\%) } \\
\hline Girassol & $33,17^{\mathrm{cAB}}$ & $34,98^{\mathrm{abB}}$ & $35,36^{\mathrm{aA}}$ & $34,99^{\mathrm{abB}}$ & $34,44^{\mathrm{bA}}$ & $33,32^{\mathrm{cA}}$ \\
\hline Soja & $32,67^{\mathrm{dB}}$ & $35,61^{\mathrm{aA}}$ & $34,70^{\mathrm{bB}}$ & $33,67^{\mathrm{cC}}$ & $34,50^{\mathrm{bA}}$ & $32,04^{\mathrm{dB}}$ \\
\hline Milho & $33,47^{\mathrm{cA}}$ & $33,12^{\mathrm{cC}}$ & $35,18^{\mathrm{abAB}}$ & $35,95^{\mathrm{aA}}$ & $34,89^{\mathrm{bA}}$ & $32,80^{\mathrm{cA}}$ \\
\hline
\end{tabular}

a, b, c (linha) - em cada óleo, médias dos tempos de fritura seguidas de mesma letra minúscula, não diferem entre si pelo teste de Tukey $(\mathrm{P}>0,05)$;

A, B (coluna) - em cada tempo de fritura, médias dos óleos seguidas de mesma letra maiúscula, não diferem entre si pelo teste de Tukey $(\mathrm{P}>0,05)$. 
Tabela 2 - Médias de Compostos Polares, Ácidos Graxos Livres e Índice de Peróxidos para cada combinação de Óleos e Tempos de Fritura.

\begin{tabular}{|c|c|c|c|c|c|c|}
\hline \multirow[t]{2}{*}{ Óleos } & \multicolumn{6}{|c|}{ Tempos de Fritura (horas) } \\
\hline & 0,5 & 2,5 & 5,0 & 7,0 & 9,5 & 12,0 \\
\hline \multicolumn{7}{|c|}{ Compostos Polares (\%) } \\
\hline Girassol & $4,60^{\mathrm{dA}}$ & $5,94^{\mathrm{cA}}$ & $6,37^{\mathrm{bcA}}$ & $6,84^{\mathrm{bAB}}$ & $7,85^{\mathrm{aA}}$ & $8,29^{\mathrm{aA}}$ \\
\hline Soja & $5,07^{\mathrm{eA}}$ & $5,89^{\mathrm{deA}}$ & $6,68^{\mathrm{cdA}}$ & $7,42^{\mathrm{bcA}}$ & $8,25^{\mathrm{abA}}$ & $8,37^{\mathrm{aA}}$ \\
\hline Milho & $4,87^{\mathrm{eA}}$ & $5,06^{\mathrm{deB}}$ & $5,56^{\mathrm{cdB}}$ & $6,42^{\mathrm{bcB}}$ & $7,04^{\mathrm{abB}}$ & $7,50^{\mathrm{aB}}$ \\
\hline \multicolumn{7}{|c|}{ Ácidos Graxos Livres (\%) } \\
\hline Girassol & $0,11^{\mathrm{cB}}$ & $0,11^{\mathrm{cB}}$ & $0,17^{\mathrm{bB}}$ & $0,22^{\mathrm{aB}}$ & $0,25^{\mathrm{aB}}$ & $0,25^{\mathrm{aB}}$ \\
\hline Soja & $0,17^{\mathrm{cA}}$ & $0,17^{\mathrm{cA}}$ & $0,25^{\mathrm{bA}}$ & $0,28^{\mathrm{bA}}$ & $0,34^{\mathrm{aA}}$ & $0,37^{\mathrm{aA}}$ \\
\hline Milho & $0,11^{\mathrm{cB}}$ & $0,14^{\mathrm{cAB}}$ & $0,20^{\mathrm{bB}}$ & $0,20^{\mathrm{bB}}$ & $0,22^{\mathrm{bB}}$ & $0,28^{\mathrm{aB}}$ \\
\hline \multicolumn{7}{|c|}{ Índice de Peróxidos (meq/kg) } \\
\hline Girassol & $0,52^{\mathrm{aA}}$ & $0,27^{\mathrm{bB}}$ & $0,27^{\mathrm{bA}}$ & $0,25^{\mathrm{bB}}$ & $0,24^{\mathrm{bAB}}$ & $0,27^{\mathrm{bA}}$ \\
\hline Soja & $0,42^{\mathrm{aB}}$ & $0,31^{\mathrm{bA}}$ & $0,26^{\mathrm{cA}}$ & $0,21^{\mathrm{eC}}$ & $0,23^{\mathrm{deB}}$ & $0,25^{\mathrm{cdA}}$ \\
\hline Milho & $0,21^{\mathrm{cC}}$ & $0,32^{\mathrm{aA}}$ & $0,23^{\mathrm{bcB}}$ & $0,33^{\mathrm{aA}}$ & $0,26^{\mathrm{bA}}$ & $0,26^{\mathrm{bA}}$ \\
\hline
\end{tabular}

a, b, c (linha) - em cada óleo, médias dos tempos de fritura seguidas de mesma letra minúscula, não diferem entre si pelo teste de Tukey $(\mathrm{P}>0,05)$;

A, B (coluna) - em cada tempo de fritura, médias dos óleos seguidas de mesma letra maiúscula, não diferem entre si pelo teste de Tukey $(\mathrm{P}>0,05)$.

de fritura. Por um lado, a viscosidade do óleo aumenta, o que contribui para aumentar a quantidade de óleo na superfície do alimento e, ainda, a tensão interfacial entre o alimento e o óleo diminui, facilitando assim, a absorção de óleo (DOBARGANES et al., 2000a).

Os óleos estudados apresentaram teores de compostos polares abaixo do limite estabelecido por legislações de alguns países, entre 24-27\%, com valores médios de 6,56\%, conforme Tabela 2 (FIRESTONE, 1991).

Tal comportamento deve-se a adição de óleo fresco, que dilui os compostos produzidos durante a fritura e repõe os antioxidantes, contribuindo assim, para diminuir a degradação do óleo (SANIBAL \& MANICINI-FILHO, 2002).

É importante considerar a composição natural em ácidos graxos saturados do produto cárneo (snacks), os quais durante o processo de fritura, passam a fazer parte do óleo, tornando-o ainda mais resistente às reações termoxidativas. Observa-se, portanto, que a alteração dos óleos está intimamente relacionada com seu grau de insaturação, ao afetar fundamentalmente os ácidos graxos insaturados.

Cella et al. (2002) estudaram o comportamento do óleo de soja refinado durante a fritura de vegetais sob temperaturas de 170 e $180^{\circ} \mathrm{C}$ com adição periódica de óleo fresco. Os valores máximos de compostos polares totais mantiveram-se entre 18 e $21 \%$ por mais de 30 horas.

Os valores de compostos polares totais aumentaram $(\mathrm{P}<0,05)$ ao longo do tempo de fritura, independentemente do tipo de óleo utilizado, demonstrando que o aquecimento, mesmo entre períodos curtos, causou aumento no teor de compostos polares, considerado prejudicial à qualidade do óleo. A partir do tempo de fritura 2,5 horas, as porcentagens de compostos polares foram mais baixas para o óleo de milho e similares entre os óleos de girassol e soja, destacando que o óleo de milho apresentou maior estabilidade oxidativa.

Uma recomendação habitual nos Estados Unidos é desprezar os óleos de fritura que possuem conteúdo de ácidos graxos livres superior a 1\% (SMITH et al., 1985), e nas indústrias que produzem carnes fritas deve ser inferior a 2\% (USDA/FSIS, 1985, citado por FIRESTONE et al., 1991). Neste estudo não se alcançaram valores superiores a $1 \%$ em nenhuma fritura. Os valores oscilaram entre $0,11 \mathrm{e}$ 0,37\%, o que indica um baixo nível de hidrólise.

Os ácidos graxos livres foram superiores durante o processo de fritura para os três óleos, indicando o desenvolvimento de reações hidrolíticas. Em relação aos 
tipos de óleo, verifica-se que o óleo de soja apresentou os maiores valores de ácidos graxos livres em todos os tempos de fritura. Jorge et al. (2005), ao estudarem as alterações físico-químicas dos óleos de girassol, milho e soja em frituras domésticas de batatas chips, encontraram no óleo de girassol valores superiores de ácidos graxos livres aos dos óleos de milho e soja, a partir do tempo de 2,5 horas de fritura. Os ácidos graxos livres são moderadamente voláteis e uma quantidade desconhecida é perdida durante o aquecimento. Níveis moderados de ácidos graxos livres parecem não estar relacionados com a qualidade inferior do alimento e os ácidos graxos livres podem sofrer oxidação e serem convertidos em outros produtos que não são medidos por métodos de titulação (TOMPKINS \& PERKINS, 2000).

Segundo os dados apresentados na Tabela 2, os óleos apresentaram comportamento instável durante o processo de fritura para o índice de peróxidos, o que pode ser explicado pelo fato de que os peróxidos se decompõem rapidamente em produtos secundários de oxidação nas temperaturas utilizadas nos processos de fritura (CUESTA et al., 1991). A evolução deste índice foi semelhante à obtida por outros pesquisadores durante frituras de batatas com óleo de soja (DAMY \& JORGE, 2003; DEL-RÉ et al., 2003). Em estudos realizados por Masson et al. (1999), empregando diferentes óleos vegetais, observaram-se também valores baixos para os índices de peróxidos, confirmando mais uma vez que este não é um bom parâmetro para avaliar a deterioração do óleo pelo calor que, durante o aquecimento, a decomposição de hidroperóxidos é mais rápida do que seu acúmulo (STEVENSON et al., 1984).

\section{CONCLUSÕES}

Os óleos analisados, girassol, soja e milho, apesar das diferenças na composição em ácidos graxos, não apresentaram em nenhuma análise valores acima dos limites recomendados em alguns países para o descarte dos mesmos, independentemente do tempo de aquecimento. Isto sugere que as condições estabelecidas no processo de fritura como tempo e temperatura de aquecimento (12 horas $\left./ 180^{\circ} \mathrm{C}\right)$, relação $\mathrm{S} / \mathrm{V}\left(0,3 \mathrm{~cm}^{-1}\right)$ e reposição de óleo novo $(27,3 \%)$ são seguras. Os óleos estudados foram adequados à fritura dos snacks, sendo o óleo de milho de maior estabilidade oxidativa.

\section{REFERÊNCIAS BIBLIOGRÁFICAS}

AMERICAN OILCHEMISTS' SOCIETY. Official methods and recommended practices of the American Oil Chemists'Society. Arlington, 1990.
AMERICAN OILCHEMISTS' SOCIETY. Official methods and recommended practices of the American Oil Chemists'Society. Champaign, 1993.

BERGER, K. G. The practice of frying. Porim TechnoL., [S.1.], v. 9, n. 5, p. 1-34, 1984

CELLA, R. C. F.; REGITANO-D’ARCE, M.A. B.; SPOTO, M. H. F. Comportamento do óleo de soja refinado utilizado em fritura por imersão com alimentos de origem vegetal. Ciência e Tecnologia de Alimentos, Campinas, v. 22, n. 2, p. 111-116, 2002.

CORSINI, M. S.; JORGE, N. Estabilidade oxidativa de óleos vegetais utilizados em frituras de mandioca palito congelada. Ciência e Tecnologia de Alimentos, Campinas, v. 26, n. 1, p. 27-32, 2006.

CUESTA, C.; SÁNCHES-MUNIZ, F. J.; HERNANDÉZ, I.; VARELA, L. S. Modificaciones de un aceite de oliva durante las frituras sucesivas de patatas: correlaciones entre distintos índices analíticos y de evaluación global de la degradación. Revista Agroquímica Tecnologia del Alimentaria, [S.1.], v. 31, n. 4, p. 523-531, 1991.

DAMY, P. C.; JORGE, N. Determinações físico-químicas do óleo de soja e da gordura vegetal hidrogenada durante o processo de fritura descontínua. Brazilian Journal Food Technology, [S.1.], v. 6, n. 2, p. 251-257, jul./dez. 2003.

DEL-RÉ, P. V.; COLTRO, A. L.; MANENTE, J. C. P. P.; MARTI, G. E.; JORGE, N. Influência da relação superfície/ volume em frituras de batata palito. Revista do Instituto Adolfo Lutz, São Paulo, v. 62, n. 3, p. 213-219, 2003.

DOBARGANES, M. C.; MÁRQUEZ-RUIZ, G.; VELASCO, $\mathrm{J}$. Interactions between fat and food during deep-frying. European Journal Lipid Science Technology, Weinheim, v. 102, p. 521-528, 2000a.

DOBARGANES, M. C.; VELASCO, J.; DIEFFENBACHER, A. Determination of polar compounds, polymerized and oxidized triacylglycerols, and diacylglycerols in oils and fats. Pure Applied Chemistry, [S.1.], v. 72, n. 8, p. 1563-1575, 2000 b.

FIRESTONE, D. Worldwide regulation of frying fats and oils. Inform, [S.1.], v. 4, n. 12, p. 1366-1371, 1993.

FIRESTONE, D.; STIER, R. F.; BLUMENTHAL, M. M. Regulation of frying fats and oils. Food Technology, Oxford, v. 45 , n. 2, p. 90-94, 1991. 
JORGE, N.; SOARES, B. P.; LUNARDI, V. M.; MALACRIDA, C. R. Alterações físico-químicas dos óleos de girassol, milho e soja em frituras. Química Nova, São Paulo, v. 28, n. 6, p. 947-951, 2005.

MAKINSON, J. H.; GREENFIELD, H.; WONG, M. L.; WILLS, R. B. H. Fat uptake during deep-fat frying of coated and incoated foods. Journal Food Composition, San Diego, v. 1, p. 93-101, 1997.

MASSON, L.; ROBERT, P.; IZAURIETA, M.; ROMERO, N.; ORTIZ, J. Fat deterioration in deep fat frying «french fries» potatoes at restaurant and food shop sector. Grasas y Aceites, Sevilla, v. 50, n. 6, p. 460-468, 1999.

PAUL, S.; MITTAL, G. S. Regulating the use of degraded oil/ fat in deep-fat/oil food frying. Critical Review in Food Science and Nutrition, Cleveland, v. 37, n. 7, p. 635-662, 1997.

POKORNY, J. Substrate influence on the frying process. Grasas y Aceites, Sevilla, v. 49, n. 3/4, p. 265-270, 1998.

POZO-DÍEZ, R. M. Estudio del proceso de fritura de alimentos frescos y congelados prefritos: comportamiento del aceite de semilla de girasol de alto contenido en ácido oleico. 1995. 338 f. Tese (Doutorado en Farmácia) - Facultad de Farmacia, Universidad de Alcalá de Henares, Alcalá de Henares, 1995.

SANIBAL, A. A. E.; MANCINI-FILHO, J. Alterações físicas, químicas e nutricionais de óleos submetidos ao processo de fritura. Food Ingred. South American, [S.I.], v. 18, p. 64-71, 2002.

SMITH, L. M.; CLIFFORD, A. J.; CREVELING, R.; HAMBLIN, C. L. Lipid content and fatty acid profiles of various deep-fat fried foods. Journal American Oil Chemistry Society, Chicago, v. 62, p. 996-999, 1985.

STEVENSON, S. G.; VAISEY-GENSER, M.; ESKIN, N. A. M. Quality control in the use of deep frying oils. Journal American Oil Chemistry Society, Chicago, v. 61, n. 6, p. 1102-1108, 1984.

TOMPKINS, C.; PERKINS, E. G. Frying performance of low-linolenic acid soybean oil. Journal American Oil Chemistry Society, Chicago, v. 77, n. 3, p. 223-229, 2000. 